\title{
INDICADORES DE EVALUACIÓN DE LOS SERVICIOS INFORMATIVOS DE LA RADIOTELEVISIÓN PÚBLICA
}

\section{Assessment indicators for public broadcasters' news services}

Francisco Campos-Freire, Xosé Soengas-Pérez y Marta Rodríguez-Castro
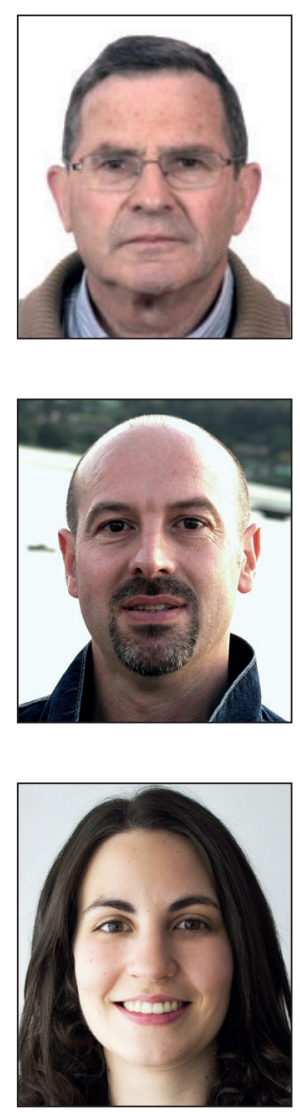

Francisco Campos-Freire es periodista, investigador y profesor de Periodismo del Departamento de Ciencias de la Comunicación de la Universidad de Santiago de Compostela (USC) desde 1991. Fue director general de la Compañía de Radiotelevisión de Galicia entre 1995-2005 y posteriormente también investigador senior del Programa Prometeo del Senescyt de Ecuador. Dentro de su línea de investigación sobre la gestión de la empresa informativa y audiovisual realizó varios estudios sobre la situación actual y tendencias del servicio audiovisual público en Europa.

https://orcid.org/0000-0003-1831-1838

francisco.campos.freire@gmail.com

Xosé Soengas-Pérez es catedrático de Comunicación Audiovisual en la Universidad de Santiago de Compostela (USC). Entre sus publicaciones destacan: Informativos radiofónicos; El tratamiento informativo del lenguaje audiovisual; El enfoque informativo. Los puntos de vista de una noticia en televisión; El papel de Internet y de las redes sociales en las revueltas árabes: una alternativa a la censura de la prensa oficial; La situación profesional de los periodistas españoles: las repercusiones de la crisis en los medios; y El ciberactivismo en el proceso de cambio político y social en los países árabes. https://orcid.org/0000-0003-3246-0477

jose.soengas@usc.es

Marta Rodríguez-Castro es graduada en Comunicación Audiovisual por la Universidad de Santiago de Compostela (USC) y máster en Investigación aplicada a medios de comunicación por la Universidad Carlos III de Madrid. Actualmente es doctoranda en Comunicación e información contemporánea en la USC y disfruta de un contrato FPU. Su investigación se centra en la gobernanza de televisión pública europea, las pruebas de valor público y los medios de proximidad.

https://orcid.org/0000-0002-1399-9154

m.rodriguez.castro@usc.es

Universidad de Santiago de Compostela Facultad de Ciencias de la Comunicación

Campus Norte. Avda. Castelao, s/n. 15782 Santiago de Compostela, España

\section{Resumen}

La reputación, la credibilidad y la rendición de cuentas son el vértice de la gobernanza de la prestación del servicio audiovisual público para sostener e incrementar su legitimidad en la sociedad actual. Para lograr este objetivo es necesario mantener y mejorar las políticas de gestión que fundamentan la misión de la radiotelevisión pública, y también desarrollar estrategias para comunicar mejor el valor social de este servicio en el contexto de la nueva ecología de medios de comunicación. Partiendo de esas premisas, este artículo aborda la necesidad y las dificultades de establecer indicadores sintéticos de evaluación de los servicios informativos como principal bloque de contenidos de la radiotelevisión pública.

\section{Palabras clave}

Indicadores; Televisión; Servicios informativos; Gobernanza; Rendición de cuentas; Calidad; Diversidad; Independencia; Pluralismo; Regulación y autorregulación.

\section{Abstract}

Reputation, credibility, and accountability are the cornerstones of governing public service media in order to sustain and increase its legitimacy in society. To achieve this objective the management policies of public service media must be maintained and enhanced according to their mission. It is also necessary to develop strategies to improve the communication of 
the social value of pubic broadcasting in the media ecosystem. On this basis, this paper discusses the need for and challenges of stabilizing assessment indicators for public broadcasters' news services.

\section{Keywords}

Indicators; Television; News services; Governance; Accountability; Quality; Diversity; Independence; Pluralism; Regulation and self-regulation.

Campos-Freire, Francisco; Soengas-Pérez, Xosé; Rodríguez-Castro, Marta (2018). “Indicadores de evaluación de los servicios informativos de la radiotelevisión pública". El profesional de la información, v. 27, n. 2, pp. 267-277.

https://doi.org/10.3145/epi.2018.mar.05

\section{Introducción}

Un sistema de indicadores compuesto o sintético permite agregar un conjunto de datos o variables simples para interpretar una realidad compleja y multidimensional en base a un modelo conceptual subyacente (Ott, 1978; OECD, 2008; Coll-Serrano et al., 2014). El propósito de este trabajo es identificar los valores, variables, tipos, precedentes y complejidad de un modelo de indicadores que pueda ser útil para evaluar la calidad de los servicios informativos como parte de la gobernanza del servicio audiovisual público. Hay que partir de los elementos que articulan la configuración del servicio audiovisual público (SAP).

La calidad es uno de sus valores esenciales (EBU, 2014) y los contenidos informativos son la columna vertebral de su programación porque encarnan uno de sus mandatos fundacionales y representan, en términos cuantitativos, un tercio de las emisiones de las cadenas principales generalistas en los países de la Unión Europea, seguidos de la ficción, el entretenimiento, la divulgación cultural, los deportes y demás géneros, según datos del Observatorio Audiovisual Europeo (EAO, 2017).

Los seis valores esenciales del servicio audiovisual público son calidad, universalidad, independencia, diversidad, innovación y rendición de cuentas

Los otros cinco aspectos que la Unión Europea de Radiodifusión considera como desiderata de los seis valores esenciales del servicio audiovisual público $(E B U, 2014)$ son universalidad, independencia, diversidad, innovación y rendición de cuentas.

Esta organización, que agrupa a 73 radiodifusores de 56 países de Europa, cuenca mediterránea y Asia occidental, estableció en 2012 un sistema de indicadores, basado en esos seis valores principales y otros 24 secundarios, para tratar de evaluar y rendir cuentas sobre la contribución a la sociedad del servicio audiovisual público.

La evaluación y la rendición de cuentas son necesarias para reforzar la legitimidad que las radiotelevisiones públicas adquirieron a través de su regulación fundacional hace cerca de cien años -la British Broadcasting Company (BBC) se constituyó en 1922- mediante la legitimación que debe reportar su contribución social para justificar su vigencia y su financiación. Esa legitimación tiene que asentarse en la percepción de su aportación al capital social (Putnam, 1993; Bourdieu, 1993) y en el reconocimiento de su valor público por parte de la sociedad (Pava; Krausz, 1997; Arriaza; Nowak; Kuhn, 2015; Powell, 2016).

La rendición de cuentas es una exigencia de control y transparencia basada en la función de la teoría de la agencia $^{1}$ de quien actúa por delegación de otro u otros (Alchian; Demsetz, 1972); es decir, de quien gobierna un servicio público en nombre del público. Es de carácter horizontal cuando se realiza desde dentro de las organizaciones y vertical cuando se hace desde fuera, desde la propia sociedad, a través de los mecanismos y medios de control social (O'Donnell, 2004).

La rendición de cuentas horizontal está más enfocada al cumplimiento de la normativa y a la comprobación cuantitativa, mientras que la segunda, que necesita los indicadores más complejos, debe rendir cuentas sobre la contribución social. La rendición de cuentas horizontal está bastante desarrollada por la regulación y corregulación a través de memorias de gestión, informes de prestación del servicio público ante parlamentos $u$ organismos reguladores, cumplimiento de convenios, auditorías internas y externas.

Pero es más compleja la rendición de cuentas vertical, porque no sólo abarca lo cualitativo de la regulación sino también la autorregulación. Estos aspectos de la rendición de cuentas se articulan de forma distinta en cada país, como estudiaron Alsius-Clavera (1996) y Eberwein et al. (2011), ampliado luego por Fengler et al. (2014) a partir del marco comparado de culturas periodísticas desarrollado por Hallin y Mancini (2004).

Además, la rendición de cuentas es imprescindible para el ejercicio de la buena gobernanza (Rodhes, 1997; Maynz, 1998; Peters; Saboie, 1980; Pierre, 2010; Głowacki, 2010; Tremblay; Brunelle, 2015), entendida como un sistema multinivel de gestión de las organizaciones que integra a todos los grupos y actores relacionados con una empresa o una institución. La gobernanza de la radiotelevisión pública está formada por el sistema de regulación, corregulación, autorregulación, representación, participación y gobierno que se relaciona con la sociedad de la que forma parte y representa.

La independencia, la universalidad y la diversidad son otros tres valores imprescindibles del servicio audiovisual público 
del siglo XXI que hay que poner en línea con la ciudadanía (Freedman; Curran, 2016).

El concepto de independencia es tan universal como ambiguo o diverso, con distintos significados a menudo contradictorios, pero no es irrelevante, por eso hay que acompañarlo de las preguntas:

- con respecto a quién: gobierno, partidos políticos, grupos de presión, fuerzas del mercado, mainstream;

- con qué medios: regulación, autorregulación, códigos éticos, financiación, competencias, etc.

La universalidad, que es una condición básica de los bienes públicos de libre acceso (Bustamante, 1999), sigue siendo un valor esencial del servicio audiovisual público como bien de interés social (Stiglitz; Rosengard, 2016), necesario en la sociedad digital actual para atender externalidades que no cubren los competidores privados (Fuchs, 2014) desde tres categorías de atributos:

- técnicas y geográficas: acceso a través de todas las tecnologías, sistemas y plataformas;

- sociales y culturales: cultura de uso común para la cohesión;

- diversidad de géneros en los contenidos de información, educación y entretenimiento.

La dimensión de la universalidad es preciso conciliarla, en el marco de renovación de la legitimidad del servicio audiovisual público, con una nueva sensibilidad hacia la ciudadanía y su cohesión social, debido a la diversidad de comunidades y culturas (Horowitz; Car, 2015) que refleja la globalización e instrumenta la fragmentación de la distribución de contenidos a través de múltiples canales y plataformas. Los valores cívico-democráticos de la sociedad en la que se desarrolla el servicio audiovisual público tienen que ser conjugados con los principios de imparcialidad, equidad y equilibrio informativo, algo que a veces no deja de provocar tensiones de antipatía o falta de afinidad ideológica entre algunas audiencias (Flood et al., 2011).

El énfasis en los valores intrínsecos (democracia, pluralismo, independencia, diversidad, ciudadanía, comunidad, identidad, contribución social, perspectiva nacional e internacional diferenciada) frente a los valores de cambio y uso (Moore, 1995; Picard, 2012; Lowe, 2016; ORF, 2016) debería marcar la diferencia entre el servicio audiovisual público y el privado. Y la calidad es el grado de eficacia y eficiencia del servicio público en el cumplimiento de los valores intrínsecos, de cambio y de uso para satisfacer las necesidades democráticas y cívicas de información, entretenimiento y formación con independencia, pluralismo, veracidad, precisión, rigor, equilibrio y neutralidad. De ahí que la evaluación y rendición de cuentas requiera más de indicadores cualitativos complejos que de impactos cuantitativos simples.

La independencia es el valor intrínseco más estrechamente relacionado con la reputación, la credibilidad y la confianza en una organización (Moe, 2010; Klimkiewicz, 2013). Depende de la gobernanza (Rodhes, 1997; Głowacki, 2010; Nowak, 2014; Tremblay; Brunelle, 2015), de la financiación (Ala-Fossi, 2012; Lowe; Berg, 2013), de la profesionalización, de sistemas de regulación y códigos de autorregulación, de la protección frente a presiones y culturas políticas de manipulación e interferencia en los medios de comunicación (Hall, 1992; Jakubowicz, 2008; Bardoel; D'Haenens, 2008; Carpentier, 2011; Nissen, 2013).

La innovación es un instrumento estratégico para renovar las organizaciones del SAP, su cultura corporativa, sus procesos y contenidos, y también contribuye para mejorar su posicionamiento, revitalización, dinamización, reputación y relación con sus públicos (Steemers, 2003; Van-Dijck; Poell, 2015; Donders; Van-den-Bulck, 2014; Głowacki; Jackson, 2016). También es importante para salvaguardar el valor social de las noticias en la era de las fuentes disruptivas (Wasserman, 2017), de la necesidad de contraste, de respuestas políticas y de alfabetización mediática crítica frente a las fake news (Tambini, 2017).

Los seis valores globales de la UER para el servicio audiovisual público del siglo XXI son pues el marco general en el que se han de desarrollar sus contenidos informativos y a los que hay que atenerse para el establecimiento de sus indicadores, teniendo en cuenta la regulación y autorregulación de su gobernanza.

\section{Metodología}

Para identificar las variables y los precedentes de un sistema de indicadores de informativos del servicio audiovisual público (SAP) necesitamos revisar las leyes de creación, memorias de cuentas, mandatos marco, planes estratégicos, contratos programa, pruebas de valor público, sistemas de gobernanza, presupuestos, memorias de cuentas o de prestación del servicio público que conforman la regulación de las radiotelevisiones públicas europeas, y también los instrumentos de autorregulación formados por códigos éticos, libros de estilo, normas editoriales, memorias de responsabilidad social corporativa (RSC), consejos de redacción y defensores de las audiencias. También identificamos y describimos otros intentos de establecimiento de indicadores globales o de desempeño del servicio audiovisual público.

A partir de esa observación construimos diversas fichas de análisis, que sintetizamos en las tablas que se reproducen más adelante, tomando como casos de estudio los cinco principales modelos estatales europeos: Alemania, Gran Bretaña, Francia, Italia y España. Seleccionamos estos países porque son los cinco de mayor población de la UE, de más alta financiación y también respectivamente representativos de los tres modelos de Hallin y Mancini (2004).

Comprobamos la correlación de la independencia con los sistemas de financiación, coste por habitante, evolución de la audiencia, desarrollo de normas éticas transparentes, definición de variables de sus libros de estilo, pruebas de valor público, evaluación de la calidad y declaración de responsabilidad social corporativa.

Cabe añadir la precisión de que un indicador es una expresión cuantitativa o cualitativa de una dimensión conceptual, cuya finalidad es mostrar o señalar algo de forma numérica, gráfica, cromática o valorativa (Coll-Serrano et al., 2012). El método más conocido para la creación de indicadores es el del decálogo establecido por la OCDE, a través de su Handbook on constructing composite indicators (OECD, 2008): 
- Desarrollo de un marco conceptual o modelo teórico.

- Selección del conjunto de variables o indicadores.

- Tratamiento estadístico de los datos.

- Análisis multivariante.

- Normalización.

- Ponderación y agregación de indicadores simples.

- Análisis de incertidumbre y sensibilidad de los indicadores compuestos.

- Relación con otros indicadores.

- Descomposición del indicador compuesto en sus indicadores simples.

- Presentación y visualización de los resultados.

\section{Tipos de indicadores}

Además de los indicadores simples y compuestos, hay otros tipos -más de 400 según la OCDE en 2008-: de desempeño, gestión, economía, valor público, RSC, eficiencia, eficacia, calidad, contexto, insumos y recursos, proceso, producto y efecto e impacto. $Y$ también se conocen distintos modelos o pruebas de análisis y de evaluación del servicio audiovisual público y de partes del mismo.

Para el ámbito de la comunicación en general, la Unesco (2008) fue de las primeras instituciones en lanzar un indicador compuesto, amplio pero complejo, para evaluar el desarrollo mediático de los países. Esta misma institución publicó unos años más tarde en Brasil otro modelo de indicadores de calidad para las emisoras públicas (Bucci; Chiaretti; Fiorini, 2012).

Las investigaciones sobre indicadores de evaluación se remontan a la segunda mitad del siglo XX, a la preocupación por la responsabilidad social de los medios y a la necesidad de trasladar a datos la rendición de cuentas sobre dichos compromisos éticos. Son los Moyens d'Assurer la Responsabilité Social ( $\mathrm{M}^{*} \mathrm{~A}^{*} \mathrm{R}^{*} \mathrm{~S}^{*}$ ) o Media Accountability Systems que proponía Bertrand (1999). Le siguen las propuestas de autores y organizaciones como Media Ethics, Media Act, Media Accountability, Media Sustainability, comparación de códigos de gobernanza, normas técnicas para medios de Global Reporting Initiative, State of the news media de Pew Research Center, el método Damian-tno de análisis de la convergencia (Nooren et al., 2014), sistemas ISO y EFQM.

La Unión Europea de Radiodifusión (EBU, 2015) elaboró en 2012 y 2015 modelos para la evaluación del SAP. El primero mediante sistema de pares parecido al de las revisiones ciegas de la producción científica, y el segundo sobre la contribución social del servicio audiovisual público, basado en indicadores de rendimiento de alcance, percepción e impacto. Este último fue puesto en práctica por televisiones del Norte y Centro de Europa (Finlandia, Suecia y Bélgica).

La BBC (2004) y ORF (2017) tienen sus propios modelos:

- el británico está basado en los valores democrático, cultural, creativo, educativo, social, comunitario y global;

- el austríaco en el valor global, formado por los valores individual, social, nacional, internacional y corporativo.

Las radiotelevisiones de Alemania, Bélgica, Noruega y Francia también emplean sistemas específicos para la evaluación cualitativa de sus servicios y la comunicación de su contribución social.
El modelo de Media Pluralism Monitor, puesto en marcha en 2013 por el Centre for Media Pluralism and Media Freedom (CMPF) del European University Institute de Florencia, evalúa anualmente los sistemas mediáticos a través de cinco áreas (protección básica, pluralismo de mercado, independencia política e inclusión social) y 20 indicadores.

El Eurobarómetro (2016) evalúa mediante una encuesta a 27.000 ciudadanos la percepción del pluralismo mediático en la UE.

El Ranking Digital Rights, una iniciativa sin ánimo de lucro creada en 2013 por Rebecca Mackinnon y New America's Open Technology Institute, analiza el cumplimiento de la responsabilidad social digital de las mega corporaciones globales a través de 35 indicadores.

En España la investigación del profesor Chaparro (Chaparro; Olmedo; Gabilondo, 2016), de la Universidad de Málaga, es pionera en la creación de un indicador sobre la rentabilidad social de la comunicación (Irscom). Para el análisis de la calidad informativa de los medios digitales Romero-Rodríguez, De-Casas-Moreno y Torres-Toukoumidis (2016) proponen un modelo estructurado basado en tres macro áreas y 75 dimensiones de indicadores, elaborados a partir del consenso de 40 expertos. Y Manfredi-Sánchez (2009) analizó los indicadores de RSC en la empresa periodística.

Los indicadores cuantitativos simples, basados tradicionalmente en la medición del contacto e impacto en las audiencias, y ahora también en el análisis de datos masivos (big data), son el instrumento fundamental de gestión del modelo de negocio del sistema audiovisual comercial, del que también dependen las radiotelevisiones públicas, a pesar de que su sistema de financiación publicitario no supera el $20 \%$ en el conjunto de la UE. Por eso el servicio audiovisual público busca nuevas estrategias y fórmulas para poder evaluar su contribución e impacto social.

Las radiotelevisiones públicas estatales de algunos países (Francia, Noruega, Finlandia, Japón o Reino Unido) evalúan su impacto a través de barómetros de calidad (Quali de France TV) o de satisfacción del servicio (Tsuji; Miyahara, 2007) para conocer las demandas y la valoración de las tarifas de canon que se pagan individualmente o a través de sus hogares. Para la televisión privada se propone el modelo de la afinidad para la evaluación de la calidad, como medida de adecuación de las noticias a los intereses de los televidentes según el índice de prioridades del barómetro mensual del CIS (López-Sanz; Medina-Laverón, 2016).

\section{Resultados del análisis de las variables}

Para reflejar la relación de complejidad de la independencia con la regulación, autorregulación y financiación presentamos a continuación seis tablas de análisis de los modelos estatales de España, Francia, Italia, Alemania y Reino Unido. Cada país tiene modelos diferentes de regulación y autorregulación de la gobernanza, algo que se constata en la existencia o no de códigos éticos, normas editoriales, uso de fact checking, pruebas de valor público, compromisos de responsabilidad social corporativa, sistemas de rendición de cuentas y de evaluación de calidad o distintos enfoques en sus libros de estilo. 
Tabla 1. Sistema de financiación y de valoración de la independencia

\begin{tabular}{|c|c|c|c|c|c|c|c|c|c|c|c|c|}
\hline \multirow[t]{2}{*}{ País } & \multirow[t]{2}{*}{$\begin{array}{l}\text { Corpora- } \\
\text { ción }\end{array}$} & \multicolumn{3}{|c|}{$\begin{array}{l}\text { Sistema de } \\
\text { financiación }\end{array}$} & \multicolumn{2}{|c|}{$\begin{array}{c}\text { Presupuesto } \\
\text { (en millones de } € \text { ) }\end{array}$} & \multicolumn{2}{|c|}{$\begin{array}{c}\text { Coste por } \\
\text { habitante }(€)\end{array}$} & \multicolumn{2}{|c|}{$\begin{array}{c}\text { Audiencia } \\
\text { cadena } \\
\text { principal (\%) }\end{array}$} & \multirow{2}{*}{$\begin{array}{l}\text { Independencia } \\
(\%) \text { según Eu- } \\
\text { robarómetro }\end{array}$} & \multirow[t]{2}{*}{$\begin{array}{c}\text { Riesgo de Inde- } \\
\text { pendencia (\%) } \\
\text { según MPM }\end{array}$} \\
\hline & & Canon & Subv. & Public. & 2010 & 2015 & 2010 & 2015 & 2014 & 2015 & & \\
\hline España & RTVE & & $x$ & $x$ & 1.200 & 952,7 & 25,81 & 18,81 & 10,0 & 9,8 & 20 & $\begin{array}{c}83 \\
\text { riesgo alto }\end{array}$ \\
\hline Francia & France TV & $x$ & & $x$ & $4.378,5$ & $4.479,6$ & 66,7 & 67,4 & 14,1 & 14,3 & 16 & $\begin{array}{c}3 \\
\text { riesgo bajo }\end{array}$ \\
\hline Italia & $R A I$ & $x$ & & $x$ & 2.821 & $2.493,1$ & 47,7 & 41,0 & 17,3 & 17 & 33 & $\begin{array}{c}75 \\
\text { riesgo alto }\end{array}$ \\
\hline Alemania & $Z D F$ & $X$ & & $x$ & 2.045 & $2.113,6$ & 25 & 26,03 & 13,3 & 12,5 & 50 & $\begin{array}{c}8 \\
\text { riesgo bajo }\end{array}$ \\
\hline $\begin{array}{l}\text { Gran } \\
\text { Bretaña }\end{array}$ & $B B C$ & $x$ & & $x$ & $7.076,1$ & $8.115,7$ & 113,2 & 125,3 & 21,7 & 24,30 & 34 & $\begin{array}{c}8 \\
\text { riesgo bajo }\end{array}$ \\
\hline
\end{tabular}

Es preciso contemplar estos aspectos para elaborar el conjunto de variables de un indicador compuesto de informativos. El sistema de financiación mediante canon, que tienen 17 países europeos a una media de 137 euros al año, es más estable e independiente que la subvención directa o la publicidad. La reducción de la financiación ha repercutido en la audiencia y en la calidad de los contenidos, como reflejan los datos de la tabla 1.

\section{La contención presupuestaria que sufre el servicio audiovisual público a nivel europeo repercute en la calidad de los informativos}

La contención presupuestaria que sufre el servicio audiovisual público a nivel europeo repercute en la calidad de los informativos al reducir coberturas, enviados especiales internacionales, atención a temas locales, supresión de periodismo de investigación e incremento de programas de plató mucho más ligeros (Freedman; Curran, 2016). La crisis económica coincide también con un cambio que registra un consumo de muchos más contenidos online (Ofcom, 2016), la mayor parte de ellos sin editar y algunos sin contrastar (fake news).
La tabla 1 muestra el contraste de la valoración de la independencia que los ciudadanos europeos encuestados hacen en cada uno de los países de sus sistemas audiovisuales públicos (Eurobarometer, 2016) con los resultados que se obtienen mediante la evaluación del Media Pluralism Monitor (CMPF, 2017). Destaca la discrepancia de Francia, donde los ciudadanos del Eurobarómetro consideran que sus medios públicos son poco independientes. Por el contrario el monitor del pluralismo mediático, basado en el análisis del sistema de regulación, le atribuye un bajo riesgo.

Dos de las radiotelevisiones analizadas, la británica y la alemana, han desarrollado una prueba de valor público, un mecanismo de regulación diseñado para decidir sobre la aprobación de nuevos servicios. En estas pruebas, vigentes en 12 países europeos, se emplean indicadores compuestos para evaluar y contraponer el valor público de un servicio -que a su vez se desglosa en múltiples variables- con respecto a su impacto sobre el mercado y la competencia.

Otro indicador relativo a la autorregulación del servicio audivisual público es la publicación de memorias de RSC, donde los propios entes analizan voluntariamente su actividad en relación con la mejora del entorno social para comunicársela a sus grupos de interés. De los países analizados, sólo Italia

Tabla 2. Sistemas de regulación y de autorregulación

\begin{tabular}{|c|c|c|c|c|c|c|c|c|c|}
\hline País & Corporación & $\begin{array}{c}\text { ¿Tiene control } \\
\text { y regulación } \\
\text { independiente? }\end{array}$ & $\begin{array}{l}\text { ¿Tiene } \\
\text { código } \\
\text { ético? }\end{array}$ & $\begin{array}{l}\text { ¿Tiene } \\
\text { libro de } \\
\text { estilo? }\end{array}$ & $\begin{array}{c}\text { ¿Tiene } \\
\text { defensor de } \\
\text { audiencias? }\end{array}$ & $\begin{array}{l}\text { ¿Tiene prue- } \\
\text { ba de valor } \\
\text { público? }\end{array}$ & $\begin{array}{l}\text { ¿Tiene } \\
\text { memoria } \\
\text { de RSC? }\end{array}$ & $\begin{array}{l}\text { ¿Tiene eva- } \\
\text { luación de la } \\
\text { calidad? }\end{array}$ & $\begin{array}{c}\text { ¿Destaca } \\
\text { uso } \\
\text { fact-check? }\end{array}$ \\
\hline España & RTVE & $\begin{array}{l}\text { Parlamento + } \\
\text { CNMV }\end{array}$ & No & Sí & $\begin{array}{l}\text { Sí (defensor del } \\
\text { espectador) }\end{array}$ & No & Sí & No & No \\
\hline Francia & France TV & CSA & No & No & $\begin{array}{l}\text { Service des } \\
\text { Relations avec } \\
\text { les Téléspecta- } \\
\text { teurs }\end{array}$ & No & Sí & No & No \\
\hline Italia & $R A I$ & $\begin{array}{l}\text { Organismo } \\
\text { de Vigilanza } \\
\text { (dependiente de } \\
\text { la } R A l \text { ) }\end{array}$ & Sí & No & No & No & No & Sí (semestral) & No \\
\hline Alemania & $Z D F$ & Fernsehrat & No & No & No & Sí & Sí & No & No \\
\hline $\begin{array}{l}\text { Gran } \\
\text { Bretaña }\end{array}$ & $B B C$ & Ofcom & Sí & Sí & Newswatch & Sí & Sí & $\begin{array}{l}\text { No de forma } \\
\text { directa, pero sí a } \\
\text { través de otros in- } \\
\text { formes periódicos } \\
\text { (Informe anual) } \\
\text { o puntuales }(A \\
\text { distinctive } B B C)\end{array}$ & No \\
\hline
\end{tabular}


Tabla 3. Mandato marco y sistema de rendición de cuentas

\begin{tabular}{|l|l|l|l|l|l|}
\hline \multicolumn{1}{|c|}{ País } & Corporación & \multicolumn{1}{|c|}{$\begin{array}{c}\text { ¿Publica quejas } \\
\text { editoriales? }\end{array}$} & $\begin{array}{c}\text { ¿Tiene mandato } \\
\text { marco? }\end{array}$ & ¿Tiene contrato programa? & \multicolumn{1}{c|}{ ¿Rinde cuentas anuales de } \\
información?
\end{tabular}

no cuenta con este tipo de indicador. En total doce radiotelevisiones públicas europeas realizan algún informe de RSC.

El sistema de regulación de la gobernanza, a través de órganos externos (parlamentos o consejos de comunicación) e internos (consejos de administración y asesores o de audiencias), así como los mecanismos de autorregulación (códigos éticos, libros de estilo, defensor de las audiencias, atención a las quejas o rendición de cuentas) son aspectos que marcan la diferencia en la calidad de la gobernanza y que tienen que ver también con la producción de informativos.

No todas las corporaciones estatales de radiotelevisión pública tienen libros de estilo o estándares similares de normas editoriales. Estos manuales son documentos elaborados a partir de las respectivas leyes de regulación, de su Royal charter $(B B C)$ o mandatos marco (documentos de estrategia que se renuevan cada 9 ó 10 años), de su contrato programa (compromisos públicos de producción) y de otros acuerdos pactados con los órganos internos y externos.
Todas las televisiones proponen medios para garantizar la presencia de los diferentes puntos de vista en las noticias, pero los procedimientos son diferentes en cada caso. En RTVE y $B B C$ se contempla la necesidad de ponderar la presencia de los sujetos informativos en relación con su relevancia o reconocimiento social; en cambio las otras tres televisiones no incluyen este requisito.

Un aspecto de consenso casi general entre las televisiones es la separación entre informacion y opinión. La RAl es la única que no mantiene esta exigencia, como se puede comprobar en la tabla 6.

\section{Conclusiones sobre la complejidad de los indicadores}

Una de las funciones y utilidades básicas de un sistema de indicadores es que permite sintetizar información para evaluar y comparar distintas realidades, en este caso televisiones y servicios informativos. No existe por el momento

Tabla 4. Tratamiento de lenguaje, imagen y fuentes en los libros de estilo

\begin{tabular}{|c|c|c|c|c|c|c|}
\hline Indicador & Variable & RTVE & France TV & RAI & $Z D F$ & $B B C$ \\
\hline \multirow{3}{*}{ Lenguaje } & ¿Mencionado? & $\begin{array}{l}\text { Lenguaje informativo } \\
\text { en televisión; lenguaje } \\
\text { radiofónico; lenguaje } \\
\text { periodístico }\end{array}$ & $\begin{array}{l}\text { "Se requiere una in- } \\
\text { formación que exige } \\
\text { rigor y precisión de } \\
\text { la lengua" }\end{array}$ & \multirow{3}{*}{$\begin{array}{l}\text { No se hacen } \\
\text { referencias }\end{array}$} & $\begin{array}{l}\text { "Comprensión"y"pre- } \\
\text { cisión lingüística" }\end{array}$ & $\begin{array}{l}\text { Desglose completo de } \\
\text { normas relativas al idioma y } \\
\text { a la terminología - Apartado } \\
\text { sobre lenguaje inapropiado }\end{array}$ \\
\hline & $\begin{array}{l}\text { Tipo de } \\
\text { mención }\end{array}$ & Directa & Indirecta & & Directa & Directa - Directa \\
\hline & $\begin{array}{l}\text { Dónde se } \\
\text { menciona }\end{array}$ & $\begin{array}{l}\text { Manual de estilo } \\
\text { (varios apartados). } \\
\text { Apartado } 6 \text { exclusivo }\end{array}$ & $\begin{array}{l}\text { Introducción apar- } \\
\text { tado } 4 .\end{array}$ & & $\begin{array}{l}\text { ¿Reciben los perio- } \\
\text { distas pautas sobre } \\
\text { cómo informar? }\end{array}$ & $\begin{array}{l}\text { News style guide - Editorial } \\
\text { guidelines (5: Daños y ofensas) }\end{array}$ \\
\hline \multirow{3}{*}{ Imagen } & ¿Mencionada? & El valor de la imagen & $\begin{array}{l}\text { Las imágenes. Nece- } \\
\text { sidad de verificación } \\
\text { antes de emisión. } \\
\text { Identificación. }\end{array}$ & \multirow{3}{*}{$\begin{array}{l}\text { No se hacen } \\
\text { referencias }\end{array}$} & \multirow{3}{*}{$\begin{array}{l}\text { No se hacen } \\
\text { referencias }\end{array}$} & $\begin{array}{l}\text { Imágenes parpadeantes, imá- } \\
\text { genes de duración muy corta }\end{array}$ \\
\hline & $\begin{array}{l}\text { Tipo de } \\
\text { mención }\end{array}$ & Directa & Directa & & & Directa \\
\hline & $\begin{array}{l}\text { Dónde se } \\
\text { menciona }\end{array}$ & $\begin{array}{l}\text { Manual de estilo } 2.1 .1 \\
(T V E)\end{array}$ & Apartado 4.3 & & & $\begin{array}{l}\text { Editorial guidelines (5: Daños } \\
\text { y ofensas) }\end{array}$ \\
\hline \multirow{3}{*}{$\begin{array}{l}\text { Calidad de } \\
\text { las fuentes }\end{array}$} & ¿Mencionada? & $\begin{array}{l}\text { Fuentes de informa- } \\
\text { ción y credibilidad } \\
\text { (identificación de las } \\
\text { fuentes) }\end{array}$ & $\begin{array}{l}\text { Calidad de las } \\
\text { fuentes }\end{array}$ & \multirow{3}{*}{$\begin{array}{l}\text { No se hacen } \\
\text { referencias }\end{array}$} & $\begin{array}{l}\text { Criterios para selec- } \\
\text { cionar expertos e } \\
\text { invitados a progra- } \\
\text { mas }\end{array}$ & $\begin{array}{l}\text { Unas buenas fuentes son } \\
\text { fundamentales. Necesidad } \\
\text { de contrastar y validar las } \\
\text { fuentes. Tratamiento con las } \\
\text { fuentes }\end{array}$ \\
\hline & $\begin{array}{l}\text { Tipo de } \\
\text { mención }\end{array}$ & Directa & Directa & & Directa & $\begin{array}{l}\text { No se hace referencia directa } \\
\text { a su calidad }\end{array}$ \\
\hline & $\begin{array}{l}\text { Dónde se } \\
\text { menciona }\end{array}$ & Manual de estilo 1.1.4 & $\begin{array}{l}\text { Apartado } 4.2 \\
\text { (Honestidad de la } \\
\text { información) }\end{array}$ & & $\begin{array}{l}\text { Procesos de decisión } \\
\text { editorial }\end{array}$ & $\begin{array}{l}\text { News style guide y Editorial } \\
\text { guide (3: Accuracy; 6: Fairness) }\end{array}$ \\
\hline
\end{tabular}


Tabla 5. Equilibrio y enfoque informativo en los libros de estilo

\begin{tabular}{|c|c|c|c|c|c|c|}
\hline Indicador & Variable & RTVE & France TV & RAI & $Z D F$ & $B B C$ \\
\hline \multirow{3}{*}{$\begin{array}{l}\text { Equilibrio } \\
\text { puntos de } \\
\text { vista }\end{array}$} & ¿Mencionado? & $\begin{array}{l}\text { El tiempo concedido para } \\
\text { el ejercicio del derecho } \\
\text { de acceso de los grupos } \\
\text { políticos y sociales } \\
\text { significativos deberá ser } \\
\text { proporcional a su arraigo, } \\
\text { implantación o reconoci- } \\
\text { miento social }\end{array}$ & $\begin{array}{l}\text { "La expresión de } \\
\text { los distintos puntos } \\
\text { de vista debe estar } \\
\text { asegurada" }\end{array}$ & $\begin{array}{l}\text { Referencia al plu- } \\
\text { ralismo en cuanto } \\
\text { a diferentes opi- } \\
\text { niones políticas, } \\
\text { sociales, culturales } \\
\text { y religiosas }\end{array}$ & $\begin{array}{l}\text { Diferentes pers- } \\
\text { pectivas sobre un } \\
\text { tema, confronta- } \\
\text { ción en entrevistas, } \\
\text { equilibrio a lo largo } \\
\text { de la programación }\end{array}$ & $\begin{array}{l}\text { Ponderación del } \\
\text { peso de cada punto } \\
\text { de vista en función } \\
\text { de su relevancia } \\
\text { social }\end{array}$ \\
\hline & $\begin{array}{l}\text { Tipo de } \\
\text { mención }\end{array}$ & Indirecta & Indirecta & Indirecta & Directa & Directa \\
\hline & $\begin{array}{l}\text { Dónde se } \\
\text { menciona }\end{array}$ & Manual de estilo, 1.1.7. & $\begin{array}{l}\text { Anexo 1, Cahier } \\
\text { des charges de FTV, } \\
\text { art. } 35\end{array}$ & $\begin{array}{l}\text { Código ético ( } 2 \text {. } \\
\text { Fundamentos } \\
\text { éticos y objetivos à } \\
\text { pluralismo) }\end{array}$ & $\begin{array}{l}\text { ¿Cómo asegura la } \\
\text { ZDF una informa- } \\
\text { ción equilibrada? } \\
\text { + Selección de } \\
\text { invitados }\end{array}$ & $\begin{array}{l}\text { Editorial guidelines } \\
\text { (4: Imparcialidad) }\end{array}$ \\
\hline \multirow[t]{3}{*}{$\begin{array}{l}\text { Tratamiento } \\
\text { formal y } \\
\text { enfoque } \\
\text { informativo }\end{array}$} & ¿Mencionado? & $\begin{array}{l}\text { Se relacionan los límites } \\
\text { en el tratamiento de la } \\
\text { información: manipula- } \\
\text { ción audiovisual, respeto } \\
\text { al anonimato, reconstruc- } \\
\text { ción de hechos, recrea- } \\
\text { ción de hipótesis y dra- } \\
\text { matizaciones; También se } \\
\text { enumeran deficiencias en } \\
\text { la cobertura informativa }\end{array}$ & $\begin{array}{l}\text { Líneas generales } \\
\text { para generar una in- } \\
\text { formación honesta }\end{array}$ & \multirow[t]{3}{*}{$\begin{array}{l}\text { No se hacen refe- } \\
\text { rencias }\end{array}$} & \multirow[t]{3}{*}{$\begin{array}{l}\text { No se hacen refe- } \\
\text { rencias }\end{array}$} & $\begin{array}{l}\text { No cuenta con una } \\
\text { referencia directa, } \\
\text { pero está latente en } \\
\text { ambos documentos }\end{array}$ \\
\hline & $\begin{array}{l}\text { Tipo de } \\
\text { mención }\end{array}$ & Indirecta & Indirecta & & & Indirecta \\
\hline & $\begin{array}{l}\text { Dónde se } \\
\text { menciona }\end{array}$ & $\begin{array}{l}\text { Manual de estilo, } 1.1 .9 \\
\text { y } 2.2\end{array}$ & Pág. 50 & & & $\begin{array}{l}\text { News Style Guide y } \\
\text { Editorial Guide }\end{array}$ \\
\hline
\end{tabular}

un modelo uniforme y estandarizado de informes de gobernanza y cumplimiento de la misión de servicio público, ni tampoco de evaluación de servicios informativos, pese a que hay modelos singulares y mucha bibliografía sobre lo que deben ser sus valores y calidad.

Existen indicadores de calidad de los informativos aceptados por muchos teóricos de la comunicación, pero no están incluidos en un documento de referencia asumido de forma integral por todas las televisiones públicas europeas. Los libros de estilo muestran objetivos comunes y al mismo tiempo reflejan las particularidades de cada emisora. Las diferencias de criterio son múltiples, principalmente cualitativas y operativas. Cada televisión tiene unos valores propios y dispone de unos procedimientos y de unos recursos particulares para garantizar la calidad, la independencia, el rigor y la pluralidad.

Aunque hay tradiciones, códigos, tratados internacionales y corrientes que traspasan fronteras, la regulación, corregulación y autorregulación del servicio audiovisual público es de competencia y carácter nacional. Por lo tanto se rige por normativas diversas y muchos aspectos que afectan al proceso informativo se abordan de forma diferente en cada país. Por eso resulta complejo homologar la calidad de los contenidos porque en cada televisión se aplican criterios propios. De hecho no todas las cadenas disponen de códigos éticos. En la mayoría de ellas muchos de los requisitos que amparan la calidad aparecen dispersos y ubicados de forma transversal en diferentes apartados de los libros de estilo.
Una normativa común y bien desarrollada facilitaría la evaluación de los informativos a partir de los mismos parámetros, garantizaría la transparencia y evitaría interferencias políticas en los contenidos, tanto a través del control permanente que permiten la mayoría de los organigramas actuales, muchas veces politizados, como en casos puntuales en los que los directivos puedan tener intereses concretos para orientar el enfoque de algún tema. Los sistemas mediáticos europeos comparados son sin duda una necesidad y un punto de partida. Pero no abundan ni es fácil actualizar sus resultados al mismo ritmo que cambian las políticas y estructuras de los mercados audiovisuales, a pesar de las dos ediciones del modelo inicial de Hallin y Mancini (2004; 2011).

Constatamos la necesidad de establecer un consenso amplio para crear un indicador que permita analizar con garantías la calidad y la rentabilidad social de las televisiones públicas, particularmente de los programas informativos. Pero también somos conscientes de la dificultad de encontrar un sistema homologable para cuantificar el grado de cumplimiento de los indicadores porque existen muchas reticencias para aceptar un protocolo de funcionamiento único. Además, la mayoría de los indicadores de calidad se construyen a partir de valores abstractos, especialmente los cualitativos, y así es muy difícil establecer unos criterios objetivos que conformen un sistema que permita medir la calidad, el rigor, la pluralidad, la transparencia y el valor social de la información. 
Tabla 6. Diversidad y tratamiento de la información política e institucional

\begin{tabular}{|c|c|c|c|c|c|c|}
\hline Indicador & Variable & RTVE & France TV & RAI & $Z D F$ & $B B C$ \\
\hline \multirow[t]{3}{*}{$\begin{array}{l}\text { Presencia de } \\
\text { diferentes grupos } \\
\text { sociales y de } \\
\text { temas de sectores } \\
\text { minoritarios }\end{array}$} & ¿Mencionada? & $\begin{array}{l}\text { Se considera legitima- } \\
\text { dos para el ejercicio del } \\
\text { derecho de acceso a } \\
\text { aquellos grupos sociales } \\
\text { y políticos significativos } \\
\text { que acrediten personali- } \\
\text { dad jurídica y represen- } \\
\text { tatividad social. }\end{array}$ & $\begin{array}{l}\text { Diferencias y diver- } \\
\text { sidad. Prevención } \\
\text { de la discriminación } \\
\text { y del odio }\end{array}$ & \multirow[t]{3}{*}{$\begin{array}{l}\text { No se hacen } \\
\text { referencias }\end{array}$} & \multirow[t]{3}{*}{$\begin{array}{l}\text { No se hacen refe- } \\
\text { rencias }\end{array}$} & $\begin{array}{l}\text { Reflejar diversidad de } \\
\text { opinión: variación ru- } \\
\text { ral-urbano, edad, nivel so- } \\
\text { cioeconómico, status quo, } \\
\text { comunidades, intereses, } \\
\text { áreas geográficas }\end{array}$ \\
\hline & $\begin{array}{l}\text { Tipo de } \\
\text { mención }\end{array}$ & Directa & Indirecta & & & Directa \\
\hline & $\begin{array}{l}\text { Dónde se } \\
\text { menciona }\end{array}$ & Manual de estilo, 1.1.7. & Pág. 41 & & & $\begin{array}{l}\text { Editorial guidelines (4: Im- } \\
\text { parcialidad; } 12 \text { : Religión) }\end{array}$ \\
\hline \multirow{3}{*}{ Información útil } & ¿Mencionada? & $\begin{array}{l}\text { Información y el interés } \\
\text { público }\end{array}$ & $\begin{array}{l}\text { Información de } \\
\text { interés público } \\
\text { (p.16) }\end{array}$ & \multirow{3}{*}{$\begin{array}{l}\text { No se hacen } \\
\text { referencias }\end{array}$} & $\begin{array}{l}\text { Criterio para } \\
\text { decidir temas in- } \\
\text { formativos: interés } \\
\text { de la audiencia }\end{array}$ & \multirow{3}{*}{$\begin{array}{l}\text { No se hace ninguna } \\
\text { referencia, pero entre los } \\
\text { valores editoriales de la } \\
B B C \text { (Editorial guidelines, } \\
\text { 1.2.6) está el servicio } \\
\text { al interés público, que } \\
\text { incluye información sobre } \\
\text { historias significativas } \\
\text { para la audiencia }\end{array}$} \\
\hline & $\begin{array}{l}\text { Tipo de } \\
\text { mención }\end{array}$ & Indirecta & $\begin{array}{l}\text { Indirecta (no habla } \\
\text { de información útil, } \\
\text { sino de interés) }\end{array}$ & & Indirecta & \\
\hline & $\begin{array}{l}\text { Dónde se } \\
\text { menciona }\end{array}$ & Manual de estilo, 1.1.1. & $\begin{array}{l}\text { Apartado 1.3.1. } \\
\text { (Oferta de progra- } \\
\text { mas) }\end{array}$ & & $\begin{array}{l}\text { Procesos de deci- } \\
\text { sión editorial }\end{array}$ & \\
\hline \multirow[t]{3}{*}{$\begin{array}{l}\text { Tratamiento de } \\
\text { la información } \\
\text { política e institu- } \\
\text { cional }\end{array}$} & ¿Mencionado? & $\begin{array}{l}\text { Información política: } \\
\text { Gobierno, actividad po- } \\
\text { lítica, ruedas de prensa } \\
\text { y declaraciones, iden- } \\
\text { tificación de políticos. } \\
\text { También normas para } \\
\text { información institu- } \\
\text { cional en "Cuestiones } \\
\text { sensibles" }\end{array}$ & $\begin{array}{l}\text { Información sobre } \\
\text { el pluralismo políti- } \\
\text { co (p71) }\end{array}$ & \multirow[t]{3}{*}{$\begin{array}{l}\text { No se hacen } \\
\text { referencias }\end{array}$} & \multirow[t]{3}{*}{$\begin{array}{l}\text { No se hacen refe- } \\
\text { rencias }\end{array}$} & $\begin{array}{l}\text { Refuerzo de la impar- } \\
\text { cialidad en periodos } \\
\text { electorales - Sección } 10 \\
\text { dedicada al tratamiento } \\
\text { de política, política públi- } \\
\text { ca y encuestas }\end{array}$ \\
\hline & $\begin{array}{l}\text { Tipo de } \\
\text { mención }\end{array}$ & Directa & Directo & & & Directa \\
\hline & $\begin{array}{l}\text { Dónde se } \\
\text { menciona }\end{array}$ & Manual de estilo 1.1.6 y 5 & Anexo & & & $\begin{array}{l}\text { Editorial guidelines (4: } \\
\text { Imparcialidad; } 10: \text { Política, } \\
\text { políticas públicas...) }\end{array}$ \\
\hline \multirow{3}{*}{$\begin{array}{l}\text { Separación infor- } \\
\text { mación/ opinión }\end{array}$} & ¿Mencionada? & $\begin{array}{l}\text { Opinión e información } \\
\text { deben estar claramente } \\
\text { delimitadas }\end{array}$ & $\begin{array}{l}\text { El principio es dis- } \\
\text { tinguir el enuncia- } \\
\text { do de los hechos, } \\
\text { por un lado, y el } \\
\text { comentario por el } \\
\text { otro. }\end{array}$ & \multirow{3}{*}{$\begin{array}{l}\text { No se hacen } \\
\text { referencias }\end{array}$} & $\begin{array}{l}\text { No es tarea de los } \\
\text { presentadores } \\
\text { evaluar eventos } \\
\text { o comunicar sus } \\
\text { opiniones perso- } \\
\text { nales. }\end{array}$ & $\begin{array}{l}\text { No emplear los términos } \\
\text { buenas/malas noticias - } \\
\text { Se permite la expresión } \\
\text { de opiniones, pero seña- } \\
\text { lándolas como tales }\end{array}$ \\
\hline & $\begin{array}{l}\text { Tipo de } \\
\text { mención }\end{array}$ & Directa & $\begin{array}{l}\text { Directa (aunque } \\
\text { breve) }\end{array}$ & & Directa & Indirecta - Directa \\
\hline & $\begin{array}{l}\text { Dónde se } \\
\text { menciona }\end{array}$ & $\begin{array}{l}\text { Manual de estilo, 1.1.2. } \\
\text { y } 3.4\end{array}$ & Pág. 66 & & $\begin{array}{l}\text { ¿Qué deben hacer } \\
\text { los presentadores } \\
\text { y los periodistas? }\end{array}$ & $\begin{array}{l}\text { News style guide - Editori- } \\
\text { al guidelines (4. Impar- } \\
\text { cialidad; } 15: \text { Conflicto de } \\
\text { interés) }\end{array}$ \\
\hline
\end{tabular}

\section{Nota}

1. La teoría de la agencia es uno de los paradigmas clásicos de la economía y la organización industrial. Se fundamenta en la relación que se establece entre la propiedad de una empresa (sea pública o privada) y los administradores o gestores de la misma a través del contrato de gobernanza y gerencia. Los agentes (gestores de las radiotelevisiones públicas) actúan y toman decisiones en nombre del principal (que es la sociedad, como propietaria del medio público) en función del contrato o encomienda del servicio, que es lo que corresponde al concepto de pacto de agencia. En tal sentido, la rendición de cuentas horizontal (interna) y vertical (externa) es fundamental para el control de la gobernanza de los bienes y servicios demaniales, es decir, de dominio público.

\section{Agradecimientos}

Los resultados de este artículo corresponden a los proyectos: Indicadores de gobernanza, financiación, rendición de cuentas, innovación, calidad y servicio público de las RTV europeas aplicables a España en el contexto digital (Referencia CSO2015-66543-P) y Usos y preferencias informativas en el nuevo mapa de medios en España: modelos de periodismo para dispositivos móviles (Ref. CSO2015-64662-C4-4-R) del Programa estatal de Fomento de la Investigación Científica y Técnica de Excelencia, subprograma estatal de Generación de Conocimiento del Ministerio de Economía y Competitividad de España, cofinanciado por el Fondo Europeo de Desarrollo Regional (Feder) de la Unión Europea. Así como de la actividad de la Red Internacional de Investigación de 
Gestión de la Comunicación (Redes 2016 G-1641 Xescom) apoyada por la Consellería de Cultura, Educación e Ordenación Universitaria de la Xunta de Galicia (Referencia ED341D R2016/019).

La autora Marta Rodríguez-Castro es beneficiaria del Programa de Formación del Profesorado Universitario (FPU16/05234) financiado por el Ministerio de Educación, Cultura y Deporte (Gobierno de España).

\section{Referencias}

Ala-Fossi, Marko (2012). "Social obsolescence of the TV fee and the financial crisis of Finnish Public Service Media". Journal of media business studies, v. 9, n. 1, pp. 33-54.

https://goo.gl/uB34Va

https://doi.org/10.1080/16522354.2012.11073535

Alchian, Armen A.; Demsetz, Harold (1972). "Production, information cost and economic organization". American economic review, v. 62, n. 5, pp. 777-795.

https://goo.gl/c5NsXH

Alsius-Clavera, Salvador (1996). Ètica i televisió informativa. Anàlisi comparativa dels codis deontològics de nou cadenes d'interès mundial. Tesis doctoral. Barcelona: Universitat Pompeu Fabra.

http://www.tdx.cat/handle/10803/7529

Arriaza, Karen; Nowak, Eva; Kuhn, Raymond (2015). Public service media in Europe. A comparative aproach. New York: Routledge-Ecrea. ISBN: 1138020680

Bardoel, Johannes; D’Haenens, Leen (2008). “Reinventing public service broadcasting in Europe: Prospects, promises and problems". Media, culture \& society, v. 30, n. 3, pp. 337-55.

https://goo.gl/U5LN49

https://doi.org/10.1177/0163443708088791

$B B C$ (2004). Building public value. Renewing the BBC for a digital world.

https://downloads.bbc.co.uk/aboutthebbc/policies/pdf/ bpv.pdf

Bertrand, Claude-Jean (1999). L'arsenal de la démocratie: Médias, déontologie et $M^{*} A^{*} R^{*} S$. París: Économica. ISBN: 2717839399

Bourdieu, Pierre (1993). Sociology in question. London: Sage. ISBN: 9780803983373

Bucci, Eugênio; Chiaretti, Marco; Fiorini, Ana-Maria (2012). Indicadores de qualidade nas emissoras públicas. Uma avaliação contemporânea. Brasília: Unesco.

http://unesdoc.unesco.org/images/0021/002166/216616por. $p d f$

Bustamante, Enrique (1999). La televisión económica. Financiación, estrategias y mercados. Barcelona: Gedisa. ISBN: 9788474327458

Carpentier, Nico (2011). Media and participation: A site of ideological-democratic struggle. Bristol: Intellect Books. ISBN: 9781841504070

https://goo.gl/hJLNxL

Chaparro, Manuel; Olmedo, Silvia; Gabilondo, Victoria (2016). "El indicador de la rentabilidad social en comunica- ción (Irscom): medir para transformar". Cuadernos de información y comunicación, v. 21, pp. 47-62.

http://revistas.ucm.es/index.php/CIYC/article/view/52944

CMPF (2017). Monitoring media pluralism in Europe: Application of the media pluralism monitor 2016 in the European Union, Montenegro and Turkey. Policy report. Centre for Media Pluralism and Media Freedom; European University Institute. http://cmpf.eui.eu/media-pluralism-monitor

Coll-Serrano, Vicente; Carrasco-Arroyo, Salvador; Blasco-Blasco, Olga; Vila-Lladosa, Luis (2012). “Design of a basic system of indicators for monitoring and evaluating Spanish cooperation's culture and development strategy". Evaluation review, v. 36, n. 4, pp. 272-302.

https://goo.gl/xsshiZ

https://doi.org/10.1177/0193841X12458104

Coll-Serrano, Vicente; Carrasco-Arroyo, Salvador; Blasco-Blasco, Olga; Vila-Lladosa, Luis (2014). "Propuesta metodológica para el diseño de un sistema de indicadores culturales local basado en la planificación estratégica". Política y sociedad, v. 51, n. 2, pp. 423-446.

https://doi.org/10.5209/rev_POSO.2014.v51.n2.42385

Donders, Karen; Van-den-Bulck, Hilde (2014). "The 'digital argument' in public service media debates: An analysis of conflicting values in Flemish management contract negotiations for VRT". En: Ferrell-Lowe, Gregory; Martin, Fiona. The value of public service media. Göteborg: Nordicom, pp. 143161. ISBN: 9789186523848

https://goo.gl/HN4Qs4

EAO (2017). Yearbook 2016 key trends. Television, cinema, video and on-demand audiovisual services-the Pan-European picture. Strasbourg: European Audiovisual Observatory. ISBN: 9789287184191

Eberwein, Tobias; Fengler, Susanne; Lauk, Epp; Leppik-Bork, Tanja (eds.) (2011). Mapping media accountability in Europe and beyond. Köln: Herbert von Halem Verlag. ISBN: 9783869620381

EBU (2014). PSM values review: The tool. European Broadcasting Union.

https://www.ebu.ch/member-services/psm-contributionsociety

EBU (2015). Public service media contribution to society. Media Intelligence Service.

https://www.ebu.ch/mis

Eurobarometer (2016). Media pluralism and democracy. Special Eurobarometer, n. 452.

http://ec.europa.eu/information_society/newsroom/ image/document/2016-47/sp452-summary_en_19666.pdf

Fengler, Susanne; Eberwein, Tobias; Mazzoleni, Gianpetro; Porlezza, Colin; Russ-Mohl, Stephan (eds.) (2014). Journalists and media accountability. An international study of news people in the digital age. New York: Peter Lang Publishing. ISBN: 9781433122811

Flood, Chris; Hutchings, Stephen; Miazhevich, Galina; Nickels, Henri (2011). Between impartiality and ideology. Journalism studies, v. 12, n. 2, pp. 221-238.

https://goo.gl/g45xME 
https://doi.org/10.1080/1461670X.2010.507934

Freedman, Des; Curran, James (2016). A future for public service television: Content and platforms in a digital world. Goldsmiths; University of London.

http://futureoftv.org.uk/report

Fuchs, Christian (2014). "Social media and the public sphere". TripleC, v. 12, n. 1, pp. 57-101.

https://www.triple-c.at/index.php/tripleC/article/view/552/529

Głowacki, Michał (2010). New public + new media $=$ new governance? The Council of Europe's approach to governance in European media.

http://ripeat.org/library/Glowacki\%20revised.pdf

Głowacki, Michał; Jackson, Lizzie (2016). "The significance of fluidity in the network age: Organisational structures within digital clusters". En: Ripe@2016 Conference: Public service media in a networked society?

Hall, Stuart (1992). "The question of cultural identity". En: Hall, Stuart; Held, David; McGrew, Tony. Modernity and its futures. Cambridge: Polity Press/Open University, pp. 274325. ISBN: 9780745609669

https://is.muni.cz/el/1421/jaro2006/PH1215/um/Hall Concepts_of_identity.pdf

Hallin, Daniel C.; Mancini, Paolo (2004). Comparing media systems. Three models of media and politics. Cambridge: Cambridge University Press. ISBN: 9780521835350 https://goo.gl/Xv12a1

Hallin, Daniel C.; Mancini, Paolo (2011). Comparing media systems beyond the Western world (Communication, society and politics). Cambridge: Cambridge University Press. ISBN: 9781107699540

Horowitz, Minna-Aslama; Car, Viktorija (2015). "The future of public service media". Medijske studije/Media studies, v. 6, n. 12, pp. 2-9.

https://hrcak.srce.hr/ojs/index.php/medijske-studije/ article/view/6149/0

Jakubowicz, Karol (2008). “Participation and partnership: A Copernican revolution to re-engineer public service media for the 21 $1^{\text {st }}$ century". En: RIPE@2008: Public service media in the 21st century: Participation, partnership and media development.

https://goo.gl/6C4akT

Klimkiewicz, Beata (2013). "Independence or balance of dependencies? Critical remarks on studying conditions of media regulators and public service media in Poland". En: Schulz, Worfgang; Valcke, Peggy; Irion, Kristina. The independence of the media and its regulatory agencies: Shedding new light on formal and actual independence against the national context. Bristol: Intellect, pp. 185-212. ISBN: 9781841507330

López-Sanz, María-Eugenia; Medina-Laverón, Mercedes (2016). "El índice de afinidad como medida de la calidad de los informativos de televisión. Estudio empírico de Antena 3 y Telecinco". Revista mediterránea de comunicación, v. 7, n. 2, pp. 141-158.

https://goo.gl/UNfxSo
Lowe, Gregory F. (2016). "What value and which values?". En: ORF. Public social value. Public value studie 2015/16. http://zukunft.orf.at/rte/upload/isabelle/orf_public_value social_studie_2016_web.pdf

Lowe, Gregory F.; Berg, Christian E. (2013). "The funding of public service media: A matter of value and values". The international journal on media management, v. 15, n. 2, pp. 77-97. https://goo.gl/fDTrfo https://doi.org/10.1080/14241277.2012.748663

Manfredi-Sánchez, Juan-Luis (2009). "Indicadores de RSC en la empresa periodística". Ámbitos, revista internacional de comunicación, v. 18, pp. 137-148.

https://idus.us.es/xmlui/handle/11441/68208

Moe, Hallvard (2010). "Governing public service broadcasting: 'Public value tests' in different national contexts". Communication, culture \& critique, v. 3, n. 2, pp. 207-223. https://goo.gl/vgL1My https://doi.org/10.1111/j.1753-9137.2010.01067.x

Moore, Mark (1995). Creating public value: Strategic management in government. London: Harvard University Press. ISBN: 9780674175587

Nissen, Christian S. (2013). “Organisational culture and structures in public media management: In search of a model for the digital age". En: Glowacki, Michael; Jackson, Lizzie. Public media management for the twenty-first century: Creativity, innovation, and interaction. London: Routledge, pp. 81-102. ISBN: 9781138653672

Nooren, Pieter; Koers, Wietske; Bangma, Menno; Berkers, Frank; Boertjes, Erik (2014). Regulation in the converged media-internet-telecom value web. Introducing the Damian method for systematic analysis of the interdependences between services, organisations and regulation. TNO Publications.

https://repository.tudelft.nl/view/tno/uuid:fbb25244-7a7d4988-9bc9-5715bde94dbc

Nowak, Eva (2014). "Between economic objectives and public remit: Positive and negative integration in European media policy". En: Donders, Karen; Pauwels, Caroline; Loisen, Jan. The Palgrave handbook of European media policy. Basingstoke: Palgrave Macmillan, pp. 96-109. ISBN: 9781 349441020

O’Donnell, Guillermo (2004): “Accountability horizontal: la institucionalización legal de la desconfianza política". Revista española de ciencia política, v. 11, pp. 11-31. https://goo.gl/EbZ9Bg

OECD (2008). Handbook on constructing composite indicators. Methodology and user guide. OECD; European Commission. ISBN: 9789264043459

http://www.oecd.org/std/42495745.pdf

Ofcom (2017). News consumption in the UK: 2016. https://goo.gl/hh5Qcb

ORF (2016). Public social value. Public value studie 2015/16. https://goo.gl/RQU6FU

ORF (2017). Public value Jahresstudie 2016/17. Der Auftrag: Bildung im digitalen zeitalter. 


\section{https://goo.gl/kURktW}

Ott, Wayne (1978). Environmental indices: Theory and practice. Ann Arbor Science Publishers. ISBN: 9780250401918

Pava, Moses L.; Krausz, Joshua (1997). "Criteria for evaluating the legitimacy of corporate social responsibility". Journal of business ethics, v. 16, n. 3, pp. 337-347.

https://goo.gl/q5j5vC

https://doi.org/10.1023/A:1017920217290

Peters, Guy B.; Saboie, Donald J. (2000). Gobernance in the twenty-first century: Revitalizing the public service. Montréal: Canadian Centre for Management Development. ISBN: 0773521291

Picard, Robert G. (2012). La creación de valor y el futuro de las empresas informativas. Por qué y cómo el periodismo debe cambiar para seguir siendo relevante en el siglo XXI. Lisboa: Media XXI. ISBN: 9789897290121

Powell, Alison (2016). "Hacking in the public interest: Authority, legitimacy, means, and ends". New media \& society, v. 18 , n. 4 , pp. $600-616$.

http://eprints.Ise.ac.uk/65380/

https://doi.org/10.1177/1461444816629470

Putnam, Robert (1993). "The prosperous community: social capital and public life". The American prospect, v. 4, n. 13, pp. 35-42.

http://prospect.org/article/prosperous-community-socialcapital-and-public-life

Rhodes, Roderick A. W. (1997). Understanding governance. Policy networks, governance, reflexivity and accountability. Buckingham: Open University Press. ISBN: 9780335197279

Romero-Rodríguez, Luis M.; De-Casas-Moreno, Patricia; Torres-Toukoumidis, Ángel (2016). "Dimensiones e indicadores de la calidad informativa en los medios digitales". Comunicar, v. 49, pp. 91-100.

https://doi.org/10.3916/C49-2016-09

Steemers, Jeanette (2003). "Public service broadcasting is not dead yet: Strategies in the $21^{\text {st }}$ century". En: Lowe, Greg- ory F.; Hujanen, Taisto. Broadcasting and convergence: New articulations of the public service remit. Göteborg: Nordicom, pp. 123-136. ISBN: 9189471180

Stiglitz, Joseph E.; Rosengard, Jay K. (2016). La economía del sector público (4a ed.). Barcelona: Bosch. ISBN: 97884 94107672

Tambini, Damian (2017). Fake news: Public policy responses. London: Media Policy Project, London School of Economics and Political Science.

http://eprints.Ise.ac.uk/73015

Tremblay, Gaëtan; Brunelle, Anne-Marie (2015). "Introduction". En: VII Colloque panaméricain en sciences de la communication. La gouvernance et service public médiatique dans les sociétés de la connaissance. Actes du Colloque. Montréal: Université du Québec. ISBN: 9782920752191 https://goo.gl/3jr8go

Tsuji, Masatsugu; Miyahara, Shoichi (2007). “Measuring the value of Japanesse public broadcasting: An application of contingent valuation method". En: Oxley, Les; Kulasiri, Don. Modsim 2007: Intl congress on modelling and simulation: Land, water and environmental management. New Zealand: Integrated Systems for Sustainability, pp. 1082-1088.

Unesco (2008). Indicadores de desarrollo mediático: marco para evaluar el desarrollo de los medios de comunicación social. París: Unesco.

http://unesdoc.UNESCO.org/images/0016/001631/163102s. $p d f$

Van-Dijck, José; Poell, Thomas (2015). “Making public television social? Public service broadcasting and the challenges of social media". Television \& new media, v. 16, n. 2, pp. 148-164.

https://goo.gl/CqyxQy

https://doi.org/10.1177/1527476414527136

Wasserman, Edward (2017). "Safeguarding the news in the era of disruptive sources". Journal of mass media ethics, v. 32, n. 2, pp. 72-85.

https://doi.org/10.1080/23736992.2017.1294020

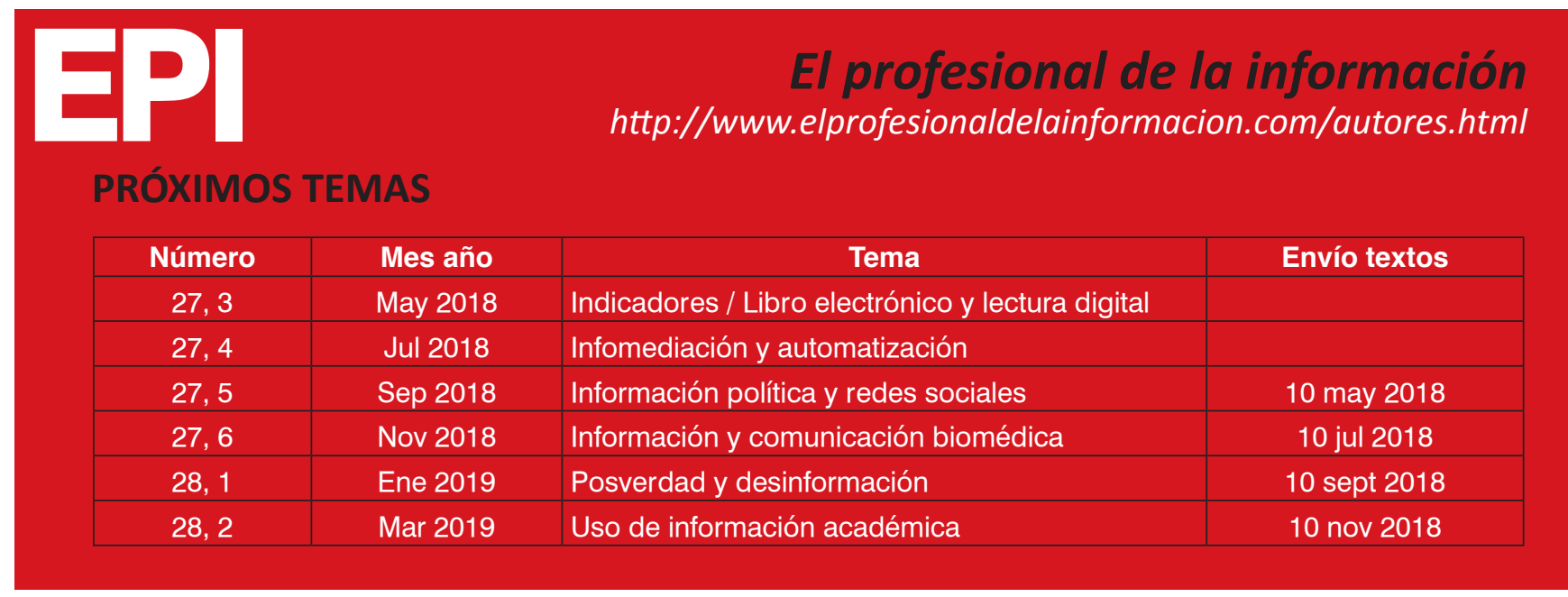

